\title{
HD-sEMG Gestures Recognition by SVM Classifier for Controlling Prosthesis
}

\author{
Hanadi Abbas Jaber ${ }^{1}$, Mofeed Turky Rashid ${ }^{2}$ \\ ${ }^{1}$ Computer Engineering Dept., Basra University, Basra, Iraq. \\ ${ }^{2}$ Electrical Engineering Dept., Basra University, Basra, Iraq. \\ hanadyce@gmail.com,mofeed.t.rashid@ieee.org
}

\begin{abstract}
Electromyography signals (EMG) are an important source to infer motion intention. It has been broadly applied in human-machine interfacing to control the neurorehabilitation devices such as prosthesis and rehabilitation robot. HD-sEMG is a muscle's activity recorded at the delimited area of the skin using $2 D$ array electrode. This strategy permits the analysis of sEMG signals in both temporal and spatial domain. Recent studies display that the spatial distribution of HD-EMG maps improves the recognition of tasks. This work investigates the use of HD-EMG recording to control upper limb prosthesis. The classification of eight hand gestures of able-bodied subjects was developed. Three feature sets were presented in this work. HOG features, time domain features(TD) and the combination of HOG and average intensity features (AIH). Combination of features possibly improved the performance of the classifier. Results show that the combined of intensity features and HOG features achieved higher performance of classifier than other features (Acc $=99.37 \%, P=98.375 \%, S=97.5 \%)$.
\end{abstract}

Index Terms-HD-sEMG, EMG pattern recognition, electrodes array, SVM classifier, spatial features component, HOG approach.

\section{INTRODUCTION}

Myoelectric prostheses employ the electromyography (EMG) signals that generated by muscle tissue and detected by surface electrodes to perform their tasks. The recorded signal used to control the movement of the robotic appendage with more actuators. These prostheses increase the abilities of amputees and other patients that suffer from physical damage or cognitive functions as a result of disease, injury, and aging.[1]

Myoelectric control is divided into two categories: pattern recognition approach and the conventional system (Direct control). In the direct control, each pair of opposite muscle site controls one motion of the prostheses. Powered artificial limbs with lots of joint movement and grasp options can cause a problem due to the existence of more degree of freedom than the control signal obtainable from the human user. Switching control is an approach to overcome this problem, whereas the patient should switch between all available joints or grasp pattern. However, switching control was impractical, not intuitive and the long time is needed to achieve a complex task.[2] Moreover, the region of muscle activity that is recorded by EMG and measured near the surface of the skin may involve the contribution of more than one muscle that causes the EMG cross-talk. The limitation of direct, switching control system and Cross-talk EMG has been a driving force for the use of pattern recognition approach to myoelectric control, which increases the number of Degree of freedom (DOF) that can be intuitively controlled.[3-5] 
Stango [6] use HD-EMG signals for controlling upper limb prostheses based on pattern recognition. Spatial features extracted from HD-EMG map making the performance of classifier robust to electrode shift with accuracy $=95 \%$ for 9 movements. Rojas [7] employ three electrodes array for Biceps, Triceps, and forearm to identify 12 gesture classes corresponding to 4 motion types with 3 effort levels. Spatial features were extracted by mean shift channel algorithm combined with intensity features obtained from five segmented map corresponds to muscles. Its classifier achieved higher performance with precision $=97.5 \%$ and sensitivity $=97.4 \%$. Geng et al. [8,9] use 2D array electrodes with 128 channel organized as a grid of $8 * 16$ matrix. Geng deal with the instantaneous recording of HD-EMG as an image. Deep convolution network was implemented for gesture recognition that classifies instantaneous sEMG images. Higher recognition accuracy reached $99.5 \%$ obtained by simple majority voting algorithm over 150 frames.

In this paper, the spatial features were proposed for gesture recognition based on two methods; the first one is the histogram oriented gradient (HOG) algorithm while the other method is the average intensity features. These features were calculated in the classification of eight hand movements of upper limb prostheses using the SVM classifier. The proposed features extraction methods satisfied the improvement of identification task. The experiments and results proved the usefulness of spatial distribution of HD-EMG data of myoelectric intensity over the muscle in the classification of tasks.

\section{Pattern Recognition Approach}

\section{A. EMG electrodes}

Electromyography (EMG) signals acquired from electrical activities of muscle by means of surface, needle or implantable electrodes. The electrode may be invasive or noninvasive, the invasive required an interface device between the patient and the robotic prostheses by surgery. Hence, surface EMG preferred to record non-invasively. The surface electrode either dry in direct connection with the patient's skin or gelled electrode that use the chemical material as the interface between skin and electrode. Recent research showed other types of surface electrodes such as multi-channel electrodes. Multi-channel electrodes use more than two channels as the linear array or 2D array of surface electrodes [10,11]. HD-sEMG channels organized in a quadrature grid of closely spaced electrodes on the skin overlying a muscle area as shown in Fig. 1.

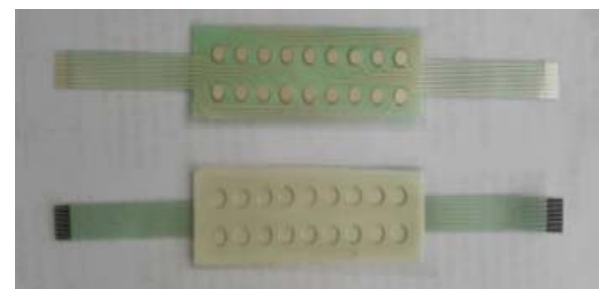

(a)

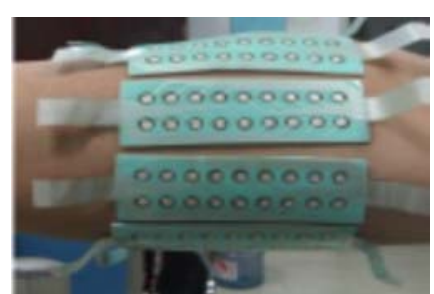

(b)

FIG .1. (A) THE HD-SEMG ELECTRODE ARRAY; (B) 8 HD-sEMG ELECTRODE ARRAYS ON THE RIGHT FOREARM [12]

\section{B. Participant}

Different types of database are available such as CSL-HDEMG database, it consists of 5 subjects performing 27 gestures. This database uses an electrode array with 192 electrodes, covering the upper forearm muscles, forming a grid of $7 \times 24$ channels. NinaPro database, a benchmark scientific database with ten electrodes on the forearm for hand 
prostheses. In the NinaPro dataset, 52 gestures were recorded for 10 trials by 27 subjects in sub-database1 (DB1) and 50 gestures were recorded for 6 trials by 40 subjects in subdatabase2 (DB2).[8]

In this study, the HD-sEMG data obtained from standard benchmark available at http:// zju-capg.org/myo/data. It consists of 128 channel prepared in matrix form 8*16 as a 2D array of closely spaced electrodes. DB-a is considered with 18 subjects. Each subject performed 8 isometric hand gesture. Each gesture repeated for 10 trials. [9]

CapgMyo database do not enforce definable contraction force when the subject performed gestures. The viewpoint of gesture recognition considered the contraction force level is a type of feature [9,12]. Identification of gesture is easier by imposing the contraction force. Further, in a real-world application, it is difficult to order users to have specific contraction force. The gestures implemented in our experiment is shown in Fig. 2.

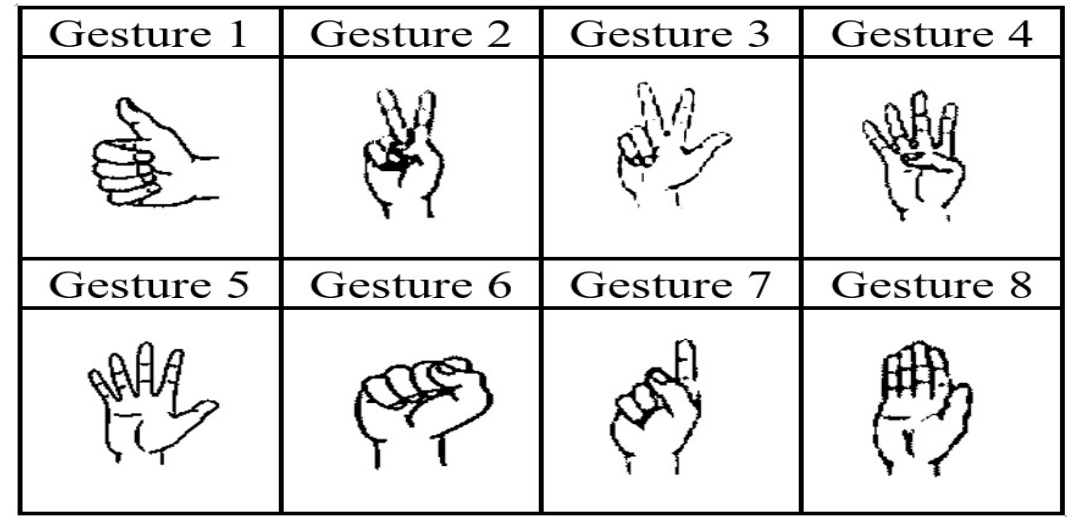

FIG. 2. HAND GESTURES CONFIGURATIONS.

\section{C. sEMG - Topography}

HD-EMG map is the spatial distribution of intensities of the active motor unit over the muscle. sEMG - map or sEMG - topography was proposed for medical application. Recently, it has been used for gesture recognition. The EMG map was calculated for a single channel as

$$
A M_{i, j}=R M S\left(s E M G_{i, j}\right)
$$

where AM is activation map of the channel (i,j), RMS is the root mean square value of EMG signal at location $\mathrm{i}, \mathrm{j}$ of $2 \mathrm{D}$ array, $\mathrm{sEMG}_{\mathrm{i}, \mathrm{j}}$ is the EMG signal at location $(\mathrm{i}, \mathrm{j})$ channel. Each pixel in the map corresponds to RMS value of a channel at location $(i, j)$ of $2 \mathrm{D}$ electrodes array.

The segmented map was calculated for several non-overlapping $200 \mathrm{~ms}$ time windows, which is the suggested window length for many studies of pattern recognition, based prosthetic control [13]. Then averaged the segmented map as

$$
\begin{aligned}
& A S M_{i, j}=\frac{1}{M} \sum_{m=1}^{M} \frac{1}{N} \sum_{n=1}^{N} S_{i j}^{2}(n) \\
& A S M_{i, j}=\frac{1}{M} \sum_{m=1}^{M} S M_{i, j}
\end{aligned}
$$

where $\mathrm{ASM}_{\mathrm{i}, \mathrm{j}}$ average segmented map located at $(\mathrm{i}, \mathrm{j})$ channel, $\mathrm{S}_{\mathrm{i}, \mathrm{j}}=\mathrm{EMG}$ signal located at $(\mathrm{i}, \mathrm{j})$ of $2 \mathrm{D}$ arrays, $\mathrm{SM}_{\mathrm{i}, \mathrm{j}}$ is the segmented map located at $(\mathrm{i}, \mathrm{j})$ channel 
$\mathrm{N}=$ total number of samples in each window of sEMG signal

$\mathrm{M}=$ number of non-overlapping windows.

\section{Feature extraction}

There are different proposal algorithms use to extract features some of them are simple such as RMS value to estimate EMG amplitude or use time domain features (TD) or more complex frequency feature such as Fourier and wavelet domain. Recent studies display that the spatial distribution of HD-EMG maps improves the recognition of tasks. The spatial features relevant to HD-EMG maps were extracted and used in identification either individual or combined so as to improve their performance [4,14- 16].

Many studies propose a non-linear relation between EMG amplitude and generated force. Therefore, the intensity features were evaluated as a common logarithm of the average intensity of HD-EMG maps [17-20].

$$
I=\log _{10} \frac{1}{N} \sum_{i, j} A M_{i, j}
$$

where $I$ is the intensity features, $A M_{i, j}$ is the intensity value of the pixel at location $(i, j)$ of activation map.

The average segmented map can be considered as images whereas each pixel corresponds to the channel. So, the problem of hand gesture can be reframed as the problem of image classification. The Histogram Oriented Gradient (HOG) algorithm is applied to extract the spatial features of the average HD-EMG map. HOG is an efficient feature extraction technique, which is widely used in image processing for the purpose of object detection. HOG counts the occurrences of gradient orientation in localized portions of an image. In this study, HOG features that extracted from the average HD-EMD map denoted as $\mathrm{H}$ features.

Combined spatial features between Intensity features and $\mathrm{H}$ features in a single feature vector by concatenation were employed. In this work, Intensity feature (I) was evaluated in a different way of Roja[10]. HD-EMG maps were calculated for each non-overlapping window in $(\mathrm{i}, \mathrm{j})$ channel and compute intensity for each window maps. Then, the average intensity for each channel. Accordingly, 128 intensity features correspond to 128 channels that combined in sequence with features result from HOG algorithm. These features were denoted as AIH (Average Intensity HOG).

Classical features may be evaluated in time, frequency and time-frequency domain. In this work, the classification was also performed using Time Domain features (TD) to compare with the two feature sets. Five TD features were computed for each channel: root means square value ( RMS), mean absolute value (MAV), the number of zero crossing (ZC), waveform length (WL) and variance (VAR). These features computed as:[21]

$$
\begin{aligned}
& R M S=\sqrt{\frac{1}{N} \sum_{n=1}^{N} x_{n}^{2}} \\
& M A V=\frac{1}{N} \sum_{n=1}^{N}\left|x_{n}\right|
\end{aligned}
$$




$$
\begin{gathered}
W L=\sum_{n=1}^{N}\left|x_{n+1}-x_{n}\right| \\
Z C=\sum_{n=1}^{N-1}\left[\operatorname{sgn}\left(x_{n} * x_{n+1}\right) \cap\left|x_{n}-x_{n+1}\right| \geq \text { thrashold }\right]
\end{gathered}
$$

$\operatorname{sgn}= \begin{cases}1 \quad x \quad \text { threshold } \\ 0 \quad \text { otherwise }\end{cases}$

$$
V A R=\frac{1}{N-1} \sum_{n=1}^{N} x_{n}^{2}
$$

Where $\mathrm{Xn}$ is the EMG signal of length $\mathrm{N}$

$\mathrm{Xn}+1$ is the advance sample value of $\mathrm{Xn}$

\section{E. Performance of classifier}

SVM classifier was applied due to its simple implementation and ease of training. Which classify 8 isometric hand gesture by finding the best hyperplane that separate data points of one class from the others. Linear kernel function gave better accuracy than other kernels.[1]

Three different SVM classifier was implemented according to different feature sets extracted from HD-sEMG data

- Classifier-based on $\mathrm{H}$ features.

- Classifier-based on the combined average intensity and $\mathrm{H}$ features denoted as (AIH).

- Classifier-based on TD features.

The performance of classifier was evaluated in term of Sensitivity (Recal) (S), Precision (P) and Accuracy (ACC) as

$$
\left.\begin{array}{c}
S=\frac{T_{P}}{T_{P}+F_{N}} \\
P=\frac{T_{P}}{T_{P}+F_{P}} \\
A c c=\frac{T_{N}+T_{P}}{T_{N}+F_{P}+T_{P}+F_{N}}
\end{array}\right\}
$$

where $T_{P}$ (true positive) is the number of samples that were correctly classified to a certain class; $\mathrm{T}_{\mathrm{N}}$ (true negative) is the number of samples that do not pertain to a certain class and were not categorized to that class; $F_{N}$ (false negative) is the number of samples pertaining to a certain class but erroneously classified into another class; Fp (false positive) is the number of samples not pertaining to a certain class but incorrectly classified into that class [18,22]. Fig. 3. Show the systematic representation of SVM classifier based AIH features. 


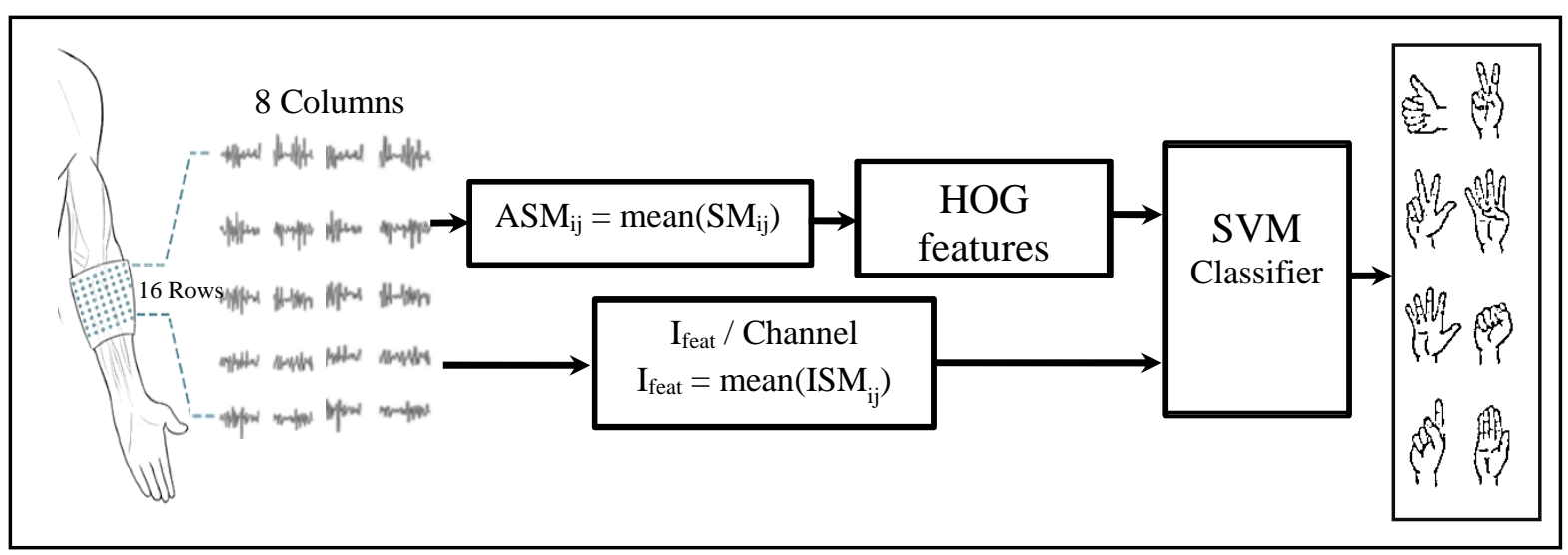

FIG .3. SCHEMATIC REPRESENTATION OF SVM CLASSIFIER BASED AIH FEATURES.

\section{III. simulation and results}

EMG signals were detected from a restricted area of the skin over the muscle using HD-sEMG electrodes. These 2D array electrodes organized in 16 rows and 8 columns. The acquired HD sEMG data were preprocessed using band-pass filtered at 20-380 $\mathrm{Hz}$ and sampled at $1000 \mathrm{~Hz}$. Each gesture recorded ten trials for each subject. For each trial, the channel was recorded for 1000 sample of instants. The identification was tested on five able-bodied subjects for the classification of eight hand gestures. The classifier was trained using the first seven trials and tested by the remaining three trails (70\% training set, 30\% test set).

In our study, two feature sets $(\mathrm{H}, \mathrm{AIH})$ were evaluated. the results illustrate the scope of each feature set to identify eight gestures. The performance of SVM classifier based $\mathrm{H}$, AIH features compared with classifier based classical methods (TD features). The performance of each gesture was averaged between five subjects and presented in term of mean and standard deviation.

Table 1. displays the results of gesture recognition based on AIH features. It can be observed from Table 1 the SVM classifier based AIH features have higher performance and achieved lower standard deviation.

TABLE 1. ACCURACY, PRECISION AND SENSITIVITY OF 8 GESTURE RECOGNITION USING AIH FEATURES AVERAGED BETWEEN FIVE SUBJECTS AND PRESENTED IN TERM OF MEAN AND STANDARD DEVIATION.

\begin{tabular}{cccc}
\cline { 2 - 4 } Gestures & \multicolumn{2}{c}{ Performance of SVM classifier based on AIH features } \\
\cline { 2 - 4 } & Accuracy $\%$ & Precision $\%$ & Sensitivity \% \\
\hline G1 & $99.16 \pm 1.86 \%$ & $100 \pm 0 \%$ & $93.3 \pm 14.9 \%$ \\
G3 & $100 \pm 0 \%$ & $100 \pm 0 \%$ & $100 \pm 0 \%$ \\
G4 & $99.16 \pm 1.86 \%$ & $95 \pm 11.18 \%$ & $100 \pm 0 \%$ \\
G5 & $100 \pm 0 \%$ & $100 \pm 0 \%$ & $100 \pm 0 \%$ \\
G6 & $100 \pm 0 \%$ & $100 \pm 0 \%$ & $86.6 \pm 29.8 \%$ \\
G7 & $98.3 \pm 3.7 \%$ & $100 \pm 0 \%$ & $100 \pm 0 \%$ \\
G8 & $98.3 \pm 3.7 \%$ & $92 \pm 17.8 \%$ & $100 \pm 0 \%$ \\
Average & $100 \pm 0 \%$ & $100 \pm 0 \%$ & $97.5 \pm 5.587$ \\
& $99.3 \pm 1.4$ & $98.375 \pm 3.6$ & \\
\hline
\end{tabular}


The performance of three SVM classifier based on different feature sets that shown in Fig. 4. As noted AIH considerably outperform all of the compared features and its performance was higher than that obtained when using the others feature sets. This is explicit in average accuracy $(98.3 \%, 99.3 \%, 98.7 \%$ for $\mathrm{H}$, AIH, TD features respectively). Moreover, the performance of TD outperforms that of $\mathrm{H}$. as well as in the average precision and sensitivity $(95 \% \pm 10 \%, 93.3 \% \pm 10.15 \%, 96.5 \% \pm 7.8 \%, 94.9 \% \pm 7.86 \%$ for $\mathrm{H}$, TD features respectively). While, precision and sensitivity for AIH was $(98.37 \% \pm 3.6 \%, 97.5 \%$ $\pm 5.587 \%$ ). A significant difference can be noticeable for precision and sensitivity based on AIH than other classifiers. Whereas, the standard deviation of precision and sensitivity decreased from $10 \%, 7.8 \%$ (for $\mathrm{H}$, TD features respectively) to $3.6 \%$ for AIH features.

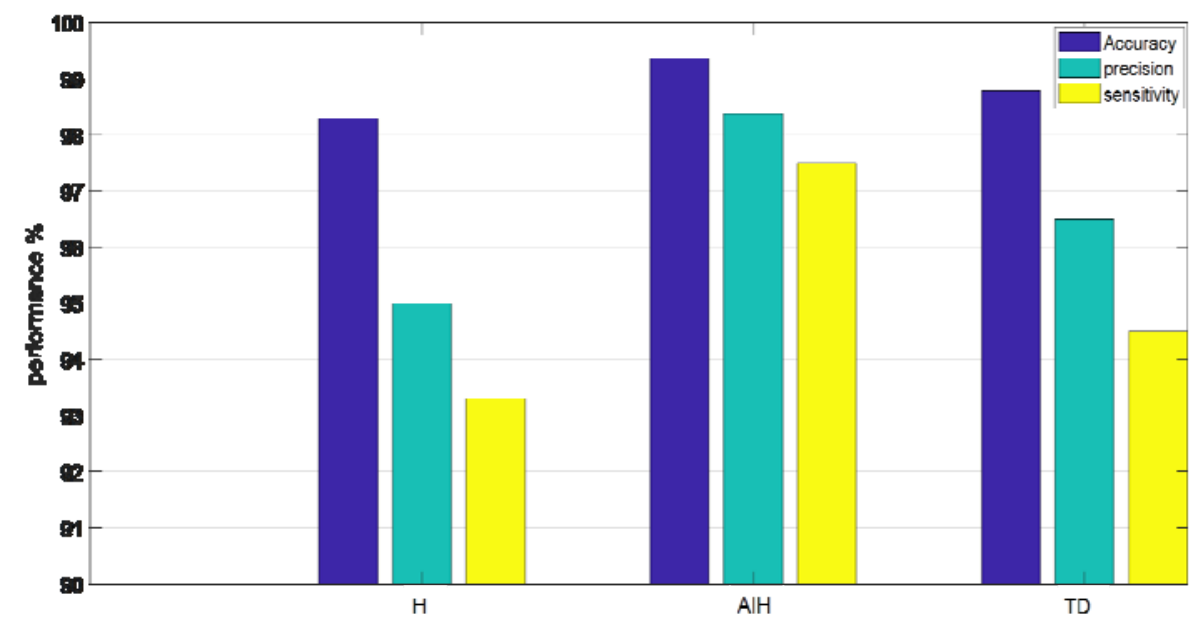

FIG . 4 THE PERFORMANCE OF SVM CLASSIFIER IN TERM OF ACCURACY, PRECISION AND SENSITIVITY BASED DIFFERENT FEATURE SETS H, AIH, TD RESPECTIVELY.

As compared with the results of Geng in [9] that used the same database (with $50 \%$ training set and $50 \%$ for testing) and the power of deep learning to classify 8 gesture DBa, the average accuracy reached $99.5 \%$. His result compared with the SVM classifier that has bad performance with nearly $18 \%$ average accuracy due to the default configuration that has a linear kernel function. While in our study a good performance for SVM classifier based AIH features, the average accuracy reached $99.37 \%$. Our result confirms that the choice of features is more important than the choice of the classifier.

Our results also compared with Rojas in [7]. Rajas used spatial features extracted by the mean shift algorithm combined with intensity feature computed in the different way of our work. Rojas segmented the HD-sEMG map of the forearm into three maps that covering the targeted muscle. Intensity feature is calculated for each map as a single feature. As a result, three intensity features are combined with mean shift features. Raja [7] used $70 \%$ as a training set and $30 \%$ for testing. Its LDA classifier achieved precision $=97.5 \%$ and sensitivity=97.4\% while in our work, SVM classifier satisfy $\mathrm{P}=98.37 \%$, S=97.5\%.

The classification accuracy of each hand gesture average between five subjects that shown in Fig. 5. As noticed G1, G2, G3, G4, G5, G8 achieved higher accuracy for AIH than other features. While G6, G7 attained higher accuracy for TD features. This is interpreting the lower standard deviation of AIH features (1.39 \%) than TD features (2.14\%).

Reduced the number of trials for training the classifier was slightly degenerate the performance. Therefore, training the SVM classifier based AIH features on the different 
number of trials from one to seven trials that shown in Fig. 6. It can be observed that accuracy of training classifier on single trial for five subjects were 98.9\%, 92.7\%, 90.6\%, 89.5\%, 91.6\% respectively with average accuracy 92.7\% which was an acceptable accuracy for one trial training. Whereas the training over three trials attained maximum performance of $97.9 \%$.

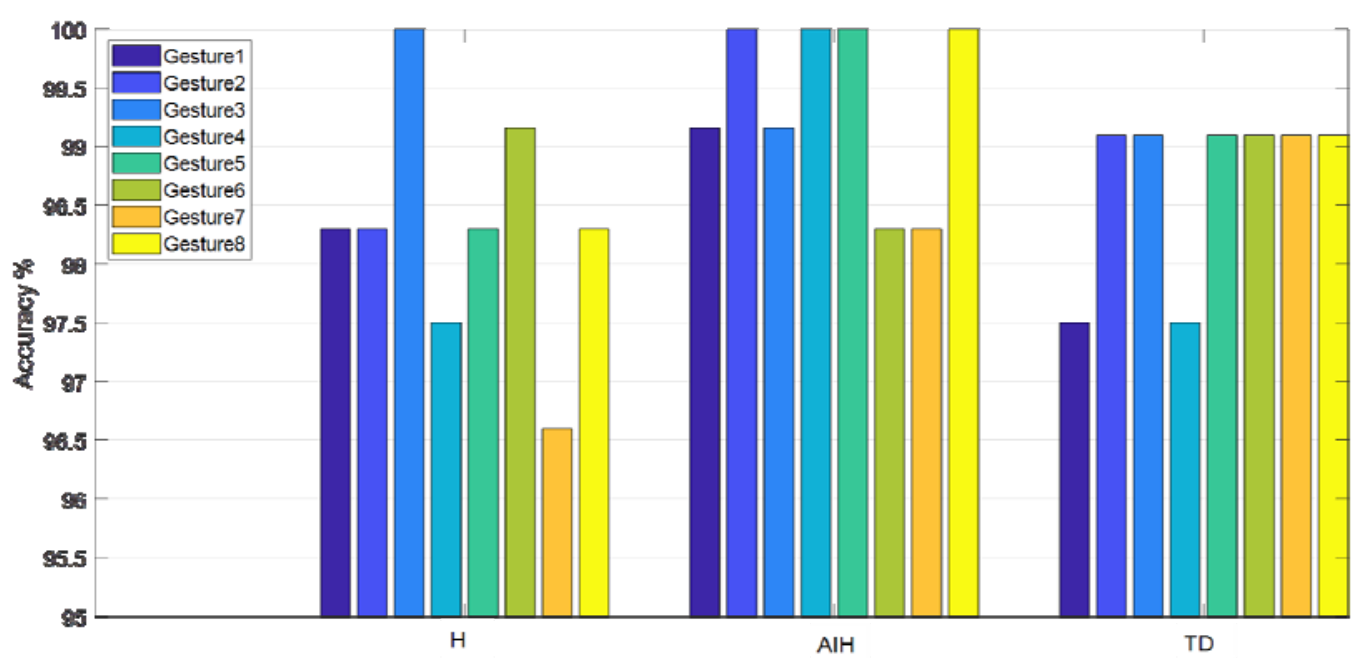

FIG. 5. CLASSIFICATION ACCURACY FOR EIGHT GESTURES BASED DIFFERENT CLASSIFIER

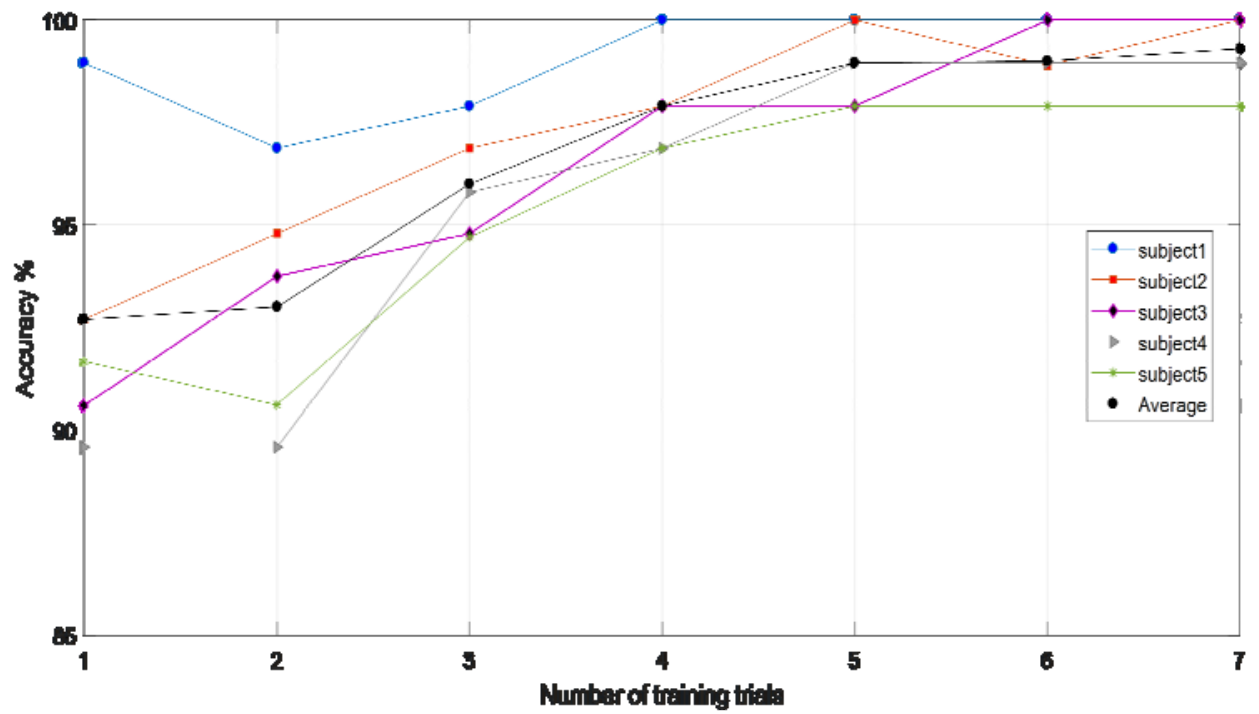

FIG .6. IDENTIFICATION ACCURACY BASED ON A DIFFERENT NUMBER OF TRAINING TRIALS.

\section{Discussion and Conclusion}

Five subjects performed eight gestures. HD-sEMG data was obtained from the standard database CapgMyo database DB-a. It consists of 128 channels configured as $8 * 16$, each channel recorded for 1000 sample instants. Each channel was divided into 5 frames of 200ms non-overlapping window. HD-EMG map was calculated for each segmented window by RMS value then averaged intensity of each segmented map per channel to get average activation map. Different feature sets were extracted $\mathrm{H}$ feature corresponds to spatial features extracted of average activation map by HOG algorithm. AIH features related to combined HOG features and intensity features. Where the intensity of each segmented window averaged to obtain average intensity for each channel as a vector its 
elements equal to the number of channels. In addition, TD features in which five features computed for each channel RMS, MAV, ZC, WL, VAR. The gesture recognized using SVM classifier based features sets. This study shows the possibility of each feature set to identify the gesture. It can be observed that the AIH achieved higher performance (ACC_AIH= $99.3 \%$, P_AIH= 98.375\%, S_AIH=97.5\%) than other feature sets. The spatial features considerably improved the classification of motion intents, this event was also presented by Rojas in [7, 18].

A good performance of the classifier should be robust to fatigue and electrode-skin contact impedance. Therefore in the future work, it is interesting to test SVM classifier based AIH features with respect to shift electrodes and fatigue. Also may be additional features correspond to frequency content can be used to mend even more the identification performance.

\section{References}

[1] P.M.Pilarski, M.R.Dawson and R.S.Sutton, " Adaptive artificial limbs, A real-time approach to prediction and anticipation", IEEE Robotics and Automation Magazine, 8 March 2013.

[2] P.M.Pilarski, M.R.Dawson, and R.S.Sutton, "Application of real-time machine learning to myoelectric prostheses control: A case series in adaptive switching", ISPO 2015.

[3] D. Farina, N. Jiang, H. Rehbaum, " The extraction of neural information from surface EMG for the control of upper limb prosthesis Emerging and challenging," IEEE Trans. Neural Syst. Rehabil. Eng. , vol. 22, no. 4, pp. $797-$ 808, July 2014.

[4] E. The scheme, K. Engllehart, "Electromyogram pattern recognition for control of powered upper limb prosthesis: state of the art and challenges for clinical use," Journal of Rehabil. Research and development, vol. 48, no. 6, pp. 643-660, 2011.

[5] N. Meselmani, M. Khrayzat, K. Chahine, "pattern recognition of EMG signals: towards adaptive control of Robotic Arm", IEEE International Multidisciplinary Conf. on Eng. Tech. 2016.

[6] A. Stango, F. Negro, D. Farina, " Spatial correlation of High-Density EMG signals provided features robust to electrode number shift in pattern recognition for My control," IEEE Trans. Neural Syst. Rehabil. Eng. , vol.23, no.2, pp.189-198, March 2015.

[7] M. Rojas, M.A. Mananas, J.F. Alonso," A novel spatial features for the identification of motor tasks using HDEMG," Sensors, 17, 1597, 2017.

[8] W. Geng, wenguang. Jin, Yu.Hu, Yu.Du, " Gesture recognition by instantaneous surface EMG images", Scientific Reports, 2016.

[9] ] W. Geng, Y.D. wenguang, y. Hu, " Surface EMG - based inter-session gesture recognition enhanced by Deep domain an adaptation," Sensors, 17, 458, 2017.

[10] J. Kilby, K. Prasad , G. Mawston, "Multi-channel surface electromyography electrodes: A Review, IEEE sensors journal, 2016.

[11] C.C. Cordova, C. Ramirez, " EMG signal pattern recognition based on feedforward artificial neural network applied to robotic prosthesis Myoelectric control," Future technology conference 2016.

[12] Phinyomark, A. Phukpattaranont, P. Limsakul, " Feature reduction and selection for EMG signal classification", Expert Syst. Appl. 39, 7420-7431, 2012.

[13] L.H. Smith, L.J. Hargrove, B.A. Lock, T.A. Kuiken, " Determining the optimal window length for pattern recognition based myoelectric control: Balancing the competing effects of classification error and controller," IEEE Trans. Neural Syst. Rehabil. Eng., vol. 19 ,no. 2 ,pp. 186-192, April 2011.

[14] Hakonen M, Piitulainen H, Visala A, "Current state of digital signal processing in myoelectric interfaces and related applications", Biomed Signal Process Control Elsevier Ltd. 18:334-59, 2015.

[15] Hargrove LJ, Englehart K, Hudgins B, " A comparison of surface and intramuscular myoelectric signal classification", IEEE Trans Biomed Eng. 54 (5),847-53, 2007.

[16] Zhang X, Zhou P. High-Density Myoelectric Pattern Recognition Toward Improved Stroke Rehabilitation. IEEE Trans Biomed Eng.;59(6):1649-57, 2012.

[17] M. Rojas, M.A. Mananas, J.F. Alonso, " Spatial distribution of HD-EMG improves identification of task and force in patients with incomplete spinal cord injury", Jor. of Neuro Eng. and Rehabil. 13; 41, 2016. 
[18] M. Rojas, M.A. Mananas, J.F. Alonso, " Prediction of isometric motor tasks and effort levels based on highdensity EMG in patients with incomplete spinal cord injury," J. Neural Eng. 13; 2016.

[19] Mañanas MA, Romero S, Topor ZL, Bruce EN, Houtz P, Caminal P," Cardiac interference in myographic signals from different respiratory muscles and levels of activity". 2001 Conf. Proc. 23rd Annu. Int. Conf. IEEE Eng. Med. Biol. Soc. 2:1115-8, 2001.

[20] Staudenmann D,RoeleveldK, Stegeman DF, van Dieen JH, "Methodological aspects of SEMG recordings for force estimation - A tutorial and review", JElectromyogr Kinesiol. ,20(3):375-87, 2010.

[21] R.H. Chowdhury, M.B.I. Reas, M.A.B.M. Ali, " Surface Electromyography signal processing and classification techniques," Sensors, 13, pp. 12431-12466, 2013.

[22] M. Rojas, M.A. Mananas, J.F. Alonso, " High-Density surface EMG maps from upper -arm and forearm muscles ", Jor. of Neuro Eng. and Rehabil. 9; 85. 2012. 